\title{
Fetal akinesia deformation sequence
}

\author{
INSERM
}

\section{Source}

INSERM. (1999). Orphanet: an online rare disease and orphan drug data base. Fetal akinesia deformation sequence. ORPHA:994

The fetal akinesia/hypokinesia sequence (or Pena-Shokeir syndrome type I) is characterized by multiple joint contractures, facial anomalies and pulmonary hypoplasia. Whatever the cause, the common feature of this sequence is decreased foetal activity. 